\title{
Precursor phenomena in frustrated systems
}

\author{
Giancarlo Franzese ${ }^{1,2,3, *}$ and Antonio Coniglio ${ }^{1,2,3,4}$ \\ ${ }^{1}$ Diportimento di Fisica “E. Amoldi”, Universitlà Roma, Tre, via Della Vasca Novole 84, I-00146 Roma, Italy \\ ${ }^{2}$ Dipartimento di Scienze Fisiche, Università di Napoli, Mostra d'Oltremare Padiglione 19, I-80125 Napoli, Italy \\ ${ }^{3}$ INFM-Unità di Napoli Mostra d'Oltremare Padiglione 19, I-80125 Napoli, Italy \\ ${ }^{4}$ INFN_Sezione di Napoli, Mostra d'Oltremare Padiglione 19, I-80125 Napoli, Italy
}

(Received 23 February 1999)

\begin{abstract}
To understand the origin of the dynamical transition, between high-temperature exponential relaxation and low-temperature nonexponential relaxation, that occurs well above the static transition in glassy systems, a frustrated spin model, with and without disorder, is considered. The model has two phase transitions, the lower being a standard spin glass transition (in the presence of disorder) or fully frustrated Ising (in the absence of disorder), and the higher being a Potts transition. Monte Carlo results clarify that in the model with (or without) disorder the precursor phenomena are related to the Griffiths (or Potts) transition. The Griffiths transition is a vanishing transition which occurs above the Potts transition and is present only when disorder is present, while the Potts transition which signals the effect due to frustration is always present. These results suggest that precursor phenomena in frustrated systems are due either to disorder and/or to frustration, giving a consistent interpretation also for the limiting cases of Ising spin glass and of Ising fully frustrated model, where also the Potts transition is vanishing. This interpretation could play a relevant role in glassy systems beyond the spin systems case. [S1063-651X(99)03106-2]
\end{abstract}

PACS number(s): 05.70.Fh, 02.70.Lq, 75.10.Nr

Experiments on glassy systems such as structural glasses, ionic conductors, supercooled liquids, polymers, colloids, and spin glasses (SG) [1] show that precursor phenomena occur at some temperature $T^{*}$ well above the static transition. In particular, the density-density or spin-spin autocorrelation function in glasses [2] or spin glasses [3] has a transition from a high-temperature exponential behavior to a low-temperature nonexponential behavior at $T^{*}$. Many attempts to relate these dynamical transitions to thermodynamic [4] or, alternatively, percolation [5,6] transitions are present in the literature, each one supported by numerical simulations [5-8]. In particular for SG models, where random distributed ferromagnetic and antiferromagnetic interactions give rise to frustration, Randeria et al. [4] suggested that $T^{*}$ should be smaller than the Griffiths temperature $T_{c}$ [9], i.e., the critical temperature of an Ising ferromagnet [10]. Intuitively the reason is that the randomness of the model allows the presence of exponentially rare large unfrustrated regions that reach an ordered state at $T_{c}$, each one with a characteristic length and a characteristic relaxation time. The distribution of relaxation times below $T_{c}$ gives rise to a nonexponential global relaxing correlation for the system, therefore $T^{*} \leqslant T_{c}$. Numerically it is found that $T^{*}$ is close to $T_{c}$ $[1,7,8]$.

It has also been suggested that a mechanism which would lead to nonexponential relaxation in frustrated systems is associated with percolation of the Fortuin-Kasteleyn-ConiglioKlein [11] (FK-CK) clusters, which can be proved to occur at a temperature $T_{p} \leqslant T_{c}$. Since it is argued that above the percolation transition the available phase space becomes rather ramified, enough to slow down the dynamics, this percolation mechanism implies that nonexponential relaxation

\footnotetext{
*Present address: Center for Polymer Studies, Boston University, 590 Commonwealth Ave., Boston, MA 02215.
}

should occur also in fully frustrated (FF) Ising systems [12] where, due to the absence of disorder, there is no Griffiths phase while the percolation temperature $T_{p}$ is finite. Recent simulations [13] on the two-dimensional (2D) and 3D FF Ising model in fact show the existence of nonexponential relaxation below a temperature equal within the numerical precision to $T_{p}$.

One might wonder whether also in spin glasses $T^{*}=T_{p}$ or instead $T^{*}=T_{c}$, since both results would satisfy Randeira's criterion. Unfortunately, it is found that $T_{p}$ is less but close to $T_{c}$ and it is very difficult numerically to locate precisely $T^{*}$ to distinguish between $T_{p}$ and $T_{c}$.

In order to better understand the role of the percolation transition and the location of $T^{*}$ in the spin glass model in this paper we consider a frustrated model in the presence of disorder, where $T_{c}$ is quite a bit larger than $T_{p}$, so that it can be easily distinguished. The numerical results allow us to conclude that $T^{*} \simeq T_{c}$. Moreover, in the absence of disorder, i.e., in the FF case (without $T_{c}$ ), the relevant role of $T_{p}$ in the dynamical transition is confirmed, giving a scenario consistent with all the previous results. In particular, the model considered here clarifies the physical meaning of $T_{p}$, relating it to a Potts transition that vanishes in the SG case and in the FF Ising case. Therefore, in the SG case there are two vanishing transitions (the Griffiths at $T_{c}$ and the Potts transition at $T_{p}$ ) in the paramagnetic phase that give rise to nonexponential relaxation. However, since $T_{c}>T_{p}$ the first transition dominates hiding the effects of the lower transition at $T_{p}$. In the FF Ising model instead there is only the vanishing transition at $T_{p}$ which now can be manifested, marking the onset of nonexponential relaxation.

Let us consider the following frustrated model with $2 s$-state spin variables whose Hamiltonian is given by

$$
H=-s J \sum_{\langle i, j\rangle}\left[\delta_{\sigma_{i} \sigma_{j}}\left(\epsilon_{i, j} S_{i} S_{j}+1\right)-2\right],
$$




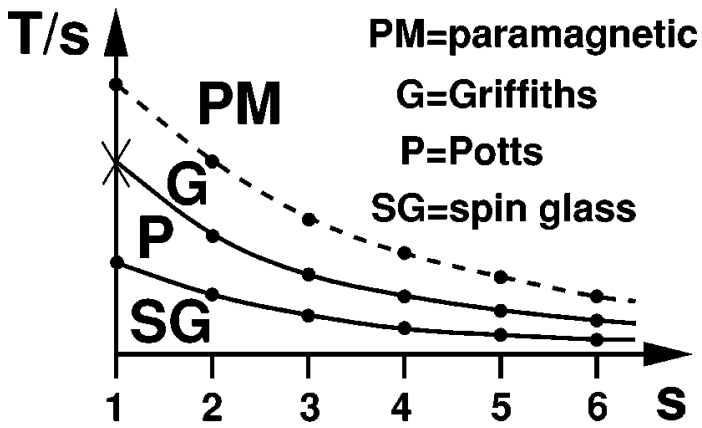

FIG. 1. Qualitative phase diagram for the PSG model in 3D as function of $s$. Solid lines mark real thermodynamic phase transitions, while dotted line marks the vanishing Griffiths transition. Cross marks Potts vanishing transition in the $s=1$ ( $\pm J$ Ising SG) case. An analogous phase diagram holds for the PFF model with a FF Ising phase at the place of the SG phase $[17,18]$ and without the $G$ phase.

where to each lattice site is associated an Ising spin $S_{i}$ $= \pm 1$ and an $s$-state Potts [14] spin $\sigma_{i}=1, \ldots, s$. The sum is extended over all nearest-neighbor sites, $J>0$ is the strength of interaction, and $\epsilon_{i, j}= \pm 1$ is a quenched variable (the sign of the interaction)

When the $\epsilon_{i, j}$ are randomly distributed, the model is a superposition of a ferromagnetic $s$-state Potts model and a $\pm J$ Ising $\mathrm{SG}$ and we will refer to it as the Potts spin glass (PSG); when the $\epsilon_{i, j}$ configuration is ordered in such a way that every elementary cell of the lattice has an odd number of -1 signs (i.e., is frustrated), the Potts model is superimposed to a FF Ising model, and we will refer to it as the Potts FF (PFF) model. For $\delta_{\sigma_{i} \sigma_{j}}=1$ (i.e., $s=1$ ) we recover, respectively, the $\pm J$ Ising SG and the FF Ising model.

Both the PSG and the PFF models exhibit [15-18], for every $s>1$, a thermodynamic transition at $T_{p}(s)$ in the same universality class of the ferromagnetic $s$-state Potts transition (see Fig. 1). This transition corresponds to the percolation transition of FK-CK clusters. For example, for $s=2$ at $T_{p}$ there is a second-order phase transition in the universality class of the Ising transition $[16,18]$. Therefore, $T_{p}$ for $s>1$ marks the Potts thermodynamic transition, which disappears for $s=1$ [19]. Both the models also exhibit a lower transition in the same universality class of the SG transition and of the FF Ising model, respectively, for the PSG [15] and the PFF model [17]. The PSG model also has a higher Griffiths temperature, at $T_{c}(s)$, which is the transition of the model without disorder, namely with all $\epsilon_{i j}=1$. It is easy to recognize that in this case the model corresponds to the ferromagnetic $2 s$ Potts model, therefore the Griffiths temperature $T_{c}(s)$ corresponds to the transition temperature of the ferromagnetic $2 s$ Potts model [14].

For $s=1$, that is, the $\pm J$ Ising SG, Ogielski [7] showed that nonexponential relaxations appear below $T_{c}$, separating the paramagnetic (PM) phase in a high-temperature PM phase and a low-temperature Griffiths ( $G$ in Fig. 1) PM phase. An analogous phase diagram holds for the 2D case, with the SG phase suppressed to $T=0$.

Here we will analyze the dynamical behavior of the $2 s$-state PSG and PFF models, with $s=2$ in 2D, simulated by standard Monte Carlo (MC) spin-flip dynamics.
PSG model. We have studied the normalized autocorrelation function

$$
f(t)=\frac{\chi_{\mathrm{SG}}(t)-\chi_{\mathrm{SG}}(t \rightarrow \infty)}{\chi_{\mathrm{SG}}(0)-\chi_{\mathrm{SG}}(t \rightarrow \infty)}
$$

of the time-dependent nonlinear susceptibility

$$
\chi_{\mathrm{SG}}(t)=\frac{1}{N} \overline{\left.\left[\sum_{i=1}^{N} S_{i}\left(t+t_{0}\right) S_{i}\left(t_{0}\right)\right]^{2}\right\rangle},
$$

where $N$ is the total number of spins, $t_{0}$ the equilibration time, $\chi_{\mathrm{SG}}(0)=N$, and where the bar stands for the average over the disorder and the angular brackets for the thermal average. Following Ref. [5] the infinite size behavior of $f(t)$ has been extrapolated at every $t$ plotting the data for finite linear sizes $L$ vs $1 / L$. To test the form of $f(t)$ we fitted the data (a) with a simple exponential, finding good fits only asymptotically for long time and for high temperatures, (b) with a stretched exponential $f_{0} \exp \left[(t / \tau)^{\beta}\right]$, finding that it fails to fit the data only for short times, and (c) with the form $f_{0} t^{-x} \exp \left[(t / \tau)^{\beta}\right]$ suggested by Ogielski [7], finding that it fits very well the data over all the time's range and the temperature's range [see Fig. 2(A)] [22]. The parameters $\beta, \tau$, and $x$ used in the fit (b) and (c) are plotted in Fig. 2(B). In both types of fit the correlation functions turn out to be nonexponential for $T \leqslant T_{c}$. The results show that $T^{*} \simeq T_{c}$. Since here the difference $T_{c}-T_{p}$ is quite large [23], it is possible to exclude $T^{*} \leqslant T_{p}[24]$.

PFF model. We have studied the autocorrelation function defined by Eqs. (2) and (3) without the average over the disorder, since there is no disorder in the model in this case. The data, shown in Fig. 3(A), are fitted with the abovementioned fitting forms $(a),(b)$, and $(c)$. The fit parameters and the integral autocorrelation time [21] are presented in Fig. 3(B) and show that the onset of nonexponential behavior is $T^{*} \simeq T_{p}$ within the numerical precision [24].

This dynamical behavior is still present in the $s=1$ case [13], where the Potts variables are not present anymore and the thermodynamic transition at $T_{p}$ disappears. If there were no frustration, the spin variables would become critical at $T_{p}$, like the Potts variables, giving rise in the free energy to two minima separated by an infinite barrier. However, due to frustration in the spin variables the two minima in the free energy will evolve into a corrugated landscape. It is this corrugated landscape which gives rise to the nonexponential behavior in the dynamics.

In the disordered case one expects that the free energy starts to appear corrugated in phase space at the Griffiths temperature $T_{c}$ due to the effect of the disorder. As the temperature is lowered at $T_{p}$ the frustration induces more roughness in the free energy landscape and preliminary numerical results [25] suggest a relation between $T_{p}$ anol the crossover for $\tau$ from a high-temperature power-law behavior to an Arrhenius behavior, well seen experimentally [26].

In conclusion, we have compared the dynamical behavior of $2 s$-state PSG and PFF models, with $s=2$. These models have two thermodynamic transitions, the lower temperature transition being a SG (or FF Ising) transition and the higher at $T_{p}$ being an Ising transition. In the disordered (PSG) model nonexponential relaxations start to appear at $T^{*}$ 

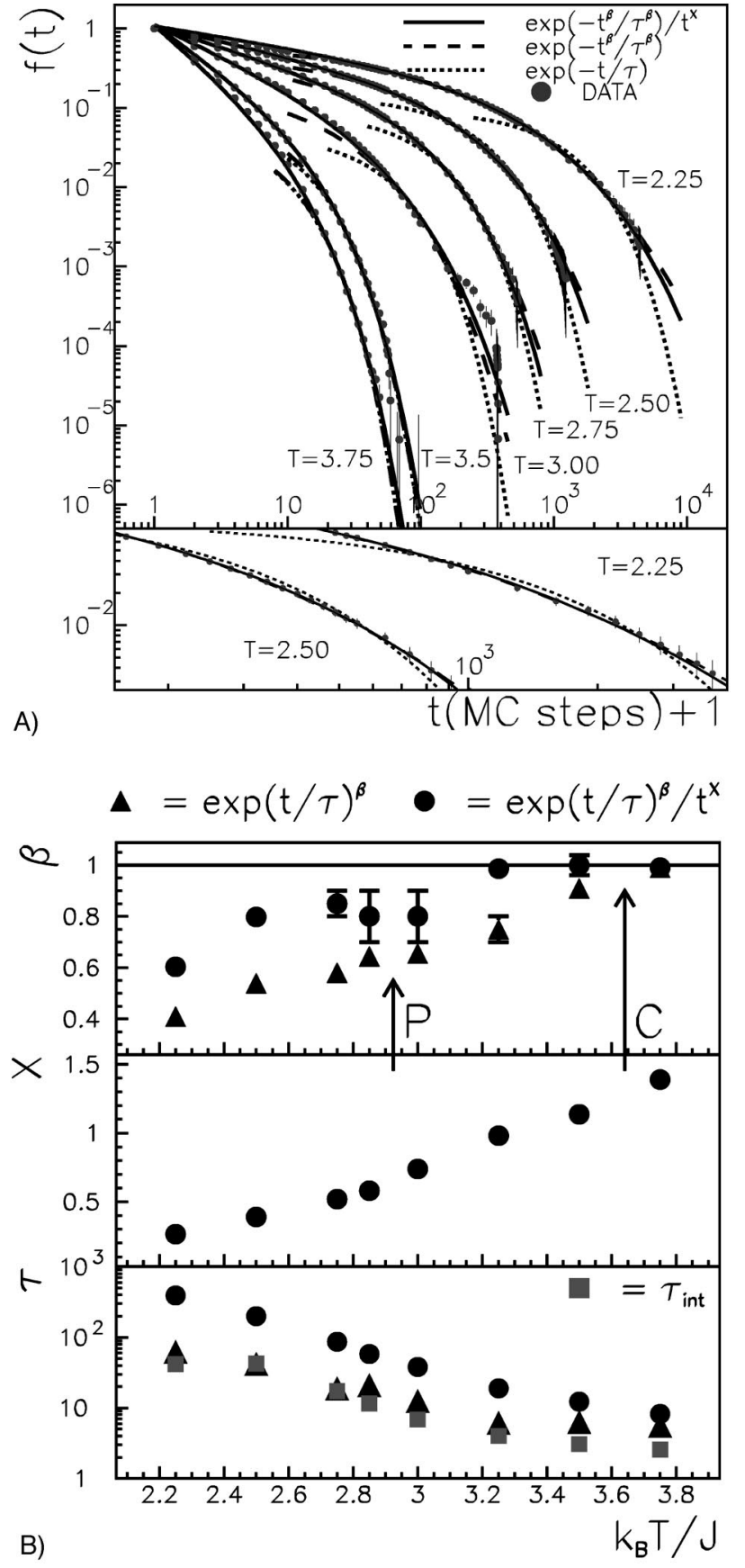

FIG. 2. PSG: (A) Nonlinear susceptibility correlation function for $L \rightarrow \infty$ limit from data for square lattices with linear sizes (in lattice steps) $L=20,24,30,40$ and periodic boundary conditions (p.b.c.) at temperature range $2.25 \leqslant T \leqslant 3.75$ (in $J / k_{B}$ unity), including $T_{c}=3.641$ [14] and $T_{p}=2.925 \pm 0.075$ [16]. Since we are well above the SG transition (occurring at $T=0$ in $2 \mathrm{D}$ ), relatively low statistics produce good data [20]. For clarity we show only some of the recorded data for some of the simulated temperatures and an enlarged view of the lower temperatures. Points are results of the simulation, solid lines the fits with the form (c) (see text), dashed lines with the form (b), and dotted lines with the form (a). Where not shown, the errors are smaller than the point's size. (B) Fit parameters used in part (A): Circles are the parameters for the form (c), triangles for the form (b); squares are the integral correlation times [21]. Arrows show $T_{p}$ and $T_{c}$.

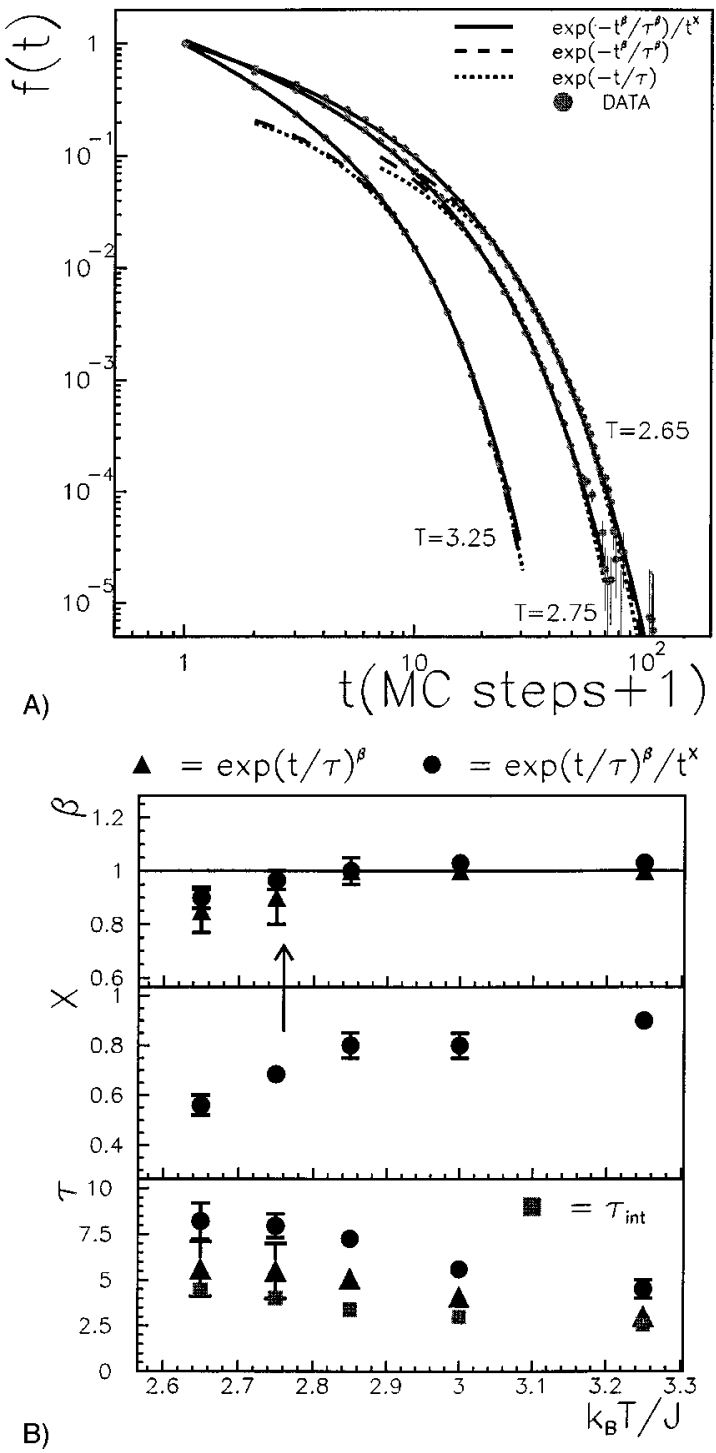

FIG. 3. PFF: (A) Nonlinear susceptibility correlation function for $L \rightarrow \infty$ limit from data for square lattices with one antiferromagnetic interaction and three ferromagnetic interactions per elementary cell, such that the systems are fully frustrated in a deterministic way, with p.b.c. and $L=20,24,30,40,50$ for the temperature range $2.65<T<3.25$, including the $T_{p}=2.73 \pm 0.03$ [18] where a secondorder Ising transition occurs. The statistics is three times that used for the PSG without the average over the disorder [20]. Only some data for some temperatures are shown. (B) Parameters used in part (A) and integral correlation times [21]. In (A) and (B) symbols are as in the preceding figure. In (B) arrow shows $T_{p}$.

$\simeq T_{c}$, i.e., at the Griffiths temperature, extending to $s=2$ the previous results for $s=1[7,8]$. In the PFF model, where there is no disorder and the Griffiths phase is not present, the effect of frustration which occurs at $T_{p}$ can be manifested. In fact, we find nonexponential relaxation starting at $T_{p}$ for both $s=2$ and $s=1$ [13], where a real and, respectively, a vanishing transition occur.

This scenario gives a consistent interpretation of all the existing results about frustrated spin systems in the literature, and could be relevant in general to understand the hightemperature behavior of glassy systems. In particular, we 
suggest that the relation of the precursor phenomena with the Griffiths phase and the Potts vanishing transition should be experimentally studied in regular crystals, such as cuprates, with doping induced disorder, where both the SG case and the FF case can be reproduced.
We would like to thank the CINECA (Bologna, Italy) for CPU time on the Cray T3D parallel computer that was used to do part of the numerical work. Partial support was given by the European TMR Network-Fractals under Contract No. FMRXCT980183.
[1] See, for example, I. A. Campbell, J. M. Flesselles, R. Jullien, and R. Botet, Phys. Rev. B 37, 3825 (1988), and references therein.

[2] C. A. Angell, Science 267, 1924 (1995).

[3] M. Mezard, G. Parisi, and M. A. Virasoro, Spin Glass Theory and Beyond (World Scientific, Singapore, 1987).

[4] M. Randeria, J. P. Sethna, and R. G. Palmer, Phys. Rev. Lett. 54, 1321 (1985); F. Cesi, C. Maes, and F. Martinelli, Commun. Math. Phys. 188, 135 (1997).

[5] I. A. Campbell and L. Bernardi, Phys. Rev. B 50, 12643 (1994).

[6] S. C. Glotzer and A. Coniglio, Comp. Mater. Sci. 4, 325 (1995).

[7] A. T. Ogielski, Phys. Rev. B 32, 7384 (1985).

[8] W. L. McMillan, Phys. Rev. B 28, 5216 (1983).

[9] R. B. Griffiths, Phys. Rev. Lett. 23, 17 (1969).

[10] $T_{c}$ is defined as the highest ordering temperature allowed by the distribution of interactions.

[11] C. M. Fortuin and P. W. Kasteleyn, Physica (Amsterdam) 57, 536 (1972); A. Coniglio and W. Klein, J. Phys. A 13, 2775 (1980).

[12] FF spin systems on lattices are such that every elementary cell of the lattice has an odd number of antiferromagnetic interactions; see J. Villain, J. Phys. C 10, 1717 (1977).

[13] A. Fierro, G. Franzese, A. de Candia, and A. Coniglio, Phys. Rev. E 59, 60 (1999).

[14] F. Wu, Rev. Mod. Phys. 54, 235 (1982).

[15] U. Pezzella and A. Coniglio, Physica A 237, 353 (1997).

[16] G. Franzese and A. Coniglio, Phys. Rev. E 58, 2753 (1998).

[17] F. di Liberto and F. Peruggi, Physica A 248, 273 (1998).

[18] G. Franzese (unpublished).

[19] For noninteger $s$ the Hamiltonian in Eq. (1) is not defined, but its partition function, written in a percolation approach [A.
Coniglio, F. di Liberto, G. Monroy, and F. Peruggi, Phys. Rev. B 44, 12605 (1991)], is well defined for every value of $s$. In this sense one can consider $s$ as a continuous variable and the $s=1$ case as the limiting case for the $s>1$ cases [14].

[20] Data are averaged over a number of random interaction configurations $n \in[8,96]$. For each temperature and each interaction configuration, after $10^{5} \mathrm{MC}$ steps (defined as the update of the whole system) of equilibration, we have recorded the data for a number of MC steps that goes from $m=3 \times 10^{5}$ for $L$ $=20$ to $m=12 \times 10^{4}$ for $L=40$, always with $n m \in\left[2 \times 10^{6}, 6\right.$ $\times 10^{6}$ ], using an annealing procedure, i.e., with a slow cooling of the system at each temperature. To verify that the statistics is sufficient for the considered temperatures, we compared the data obtained starting from a random configuration with those obtained from the annealing method, observing the same results.

[21] The integral correlation time is defined as $\tau_{\text {int }}=\lim _{t_{\max } \rightarrow \infty}\left[\frac{1}{2}\right.$ $\left.+\sum_{t=0}^{t_{\max }} f(t)\right]$.

[22] In the case (c) it is possible to do fits with only two free parameters, fitting linearly the short time behavior (described by $f_{0}$ and $x$ ) and separately the long time behavior (described by $\beta$ and $\tau$ ) with $f_{0}$ and $x$ fixed.

[23] In $2 \mathrm{D}, T_{p} /(2 s a)=1 / \ln (1+\sqrt{2 s a})$ with $a=0.0803 \pm 0.003$ [16] while $T_{c} /(2 s)=1 / \ln (1+\sqrt{2 s})$ [14], therefore $T_{c}-T_{p}$ increases with $s$.

[24] The same qualitative results are seen for $\beta$ and $x$ fit parameters associated to autocorrelation functions of the Potts magnetization, while for this critical quantity the $\tau$ exponent diverges near the transition temperature $T_{p}$.

[25] G. Fromzese and A. Coniglio (unpublished).

[26] L. Andreozzi, M. Giorolono, and D. Leporini, J. Non-Cryst. Solids 235-237, 219 (1998). 